\title{
WEIGHTED NORM INEQUALITIES FOR BILINEAR FOURIER MULTIPLIER OPERATORS
}

\begin{abstract}
GuOEN Hu
Abstract. In this paper, by kernel estimates of the bilinear Fourier multiple operator and the weighted theory for the bilinear singular integral operators, some weighted norm inequality with general weights are established for the biilinear Fourier multiplier operators.

Mathematics subject classification (2010): 42B15, 42B20.

Keywords and phrases: Bilinear Fourier multiplier, weighted estimate, Sobolev regularity, bilinear
\end{abstract} singular integral.

\section{REFERENCES}

[1] T. A. BUI AND X. T. DUONG, Weighted norm inequalities for multilinear operators and applications to multilinear Fourier multipliers, Bull. Sci. Math. 137 (2013), 63-75.

[2] M. Carrozza and A. Pass arelli Di Napoli, Composition of maximal operators, Publ. Mat. 40 (1996), 397-409.

[3] R. R. CoIfMAN And Y. Meyer, On commutators of singular integrals and bilinear singular integrals, Trans. Amer. Math. Soc. 212 (1975), 315-331.

[4] R. R. Coifman And Y. Meyer, Au delà des opérateurs pseudo-différentiels, Astériaque 57 (1978), $1-185$.

[5] M. Fujita AND N. Tomita, Weighted norm inequalities for multilinear Fourier multipliers, Trans Amer Math Soc. 364 (2012), 6335-6353.

[6] L. Grafakos, Classical Fourier analysis, GTM249, 2nd Edition, Springer, New York, 2008.

[7] L. GRAFAKOS AND N. KALtON, Multilinear Calderón-Zygmund operators on Hardy spaces, Collect. Math. 52 (2001), 169-179.

[8] L. Grafakos, A. MiYachi And N. Tomita, On multilinear Fourier multipliers of limited smoothness, Canad. J. Math. 65 (2013), 299-330.

[9] L. GRafakos And Z. Si, The Hörmander multiplier theorem for multilinear operators, J. Reine Angewandte Math. 668 (2012), 133-147.

[10] L. Grafakos and R. Torres, Multilinear Calderón-Zygmund theory, Adv. Math., 165 (2002), $124-164$.

[11] G. HU. AND C. LIN, Weighted norm inequalities for multilinear singular integral operators and applications, Anal. Appl., to appear, arXiv:1208.6346.

[12] D. S. KURTZ AND R. L. WhEedEN, Results on weighted norm inequalities for multipliers, Trans. Amer. Math. Soc. 255 (1979), 343-362.

[13] A. LERNER, Weighted norm inequalities for the local sharp maximal function, J. Fourier Anal. Appl. 10 (2004), 645-674.

[14] A. Lerner, S. Ombrossi, C. Perez, R. H. Torres and R. Trojillo-Gonzalez, New maximal functions and multiple weights for the multilinear Calderón-Zygmund theorey, Adv. Math. 220 (2009), 1222-1264.

[15] A. Miyachi And N. Tomita, Minimal smoothness conditions for bilinear Fourier multiplier, Rev Mat Iberomericana, to appear.

[16] C. PÉREZ, Weighted norm inequalities for singular integral operators, J. London Math. Soc. 49 (1994), 296-308. 
[17] C. PÉREZ, On sufficient conditions for the boundedness of the Hardy-Littlewood maximal operator between weighted $L^{p}$-spaces with different weights, Proc. London Math. Soc. 49 (1995), 135-157.

[18] N. Tomita, A Hörmander type multiplier theorem for multilinear operator, J. Func. Anal. 259 (2010), 2028-2044. 